\title{
Desenvolvendo o raciocínio matemático: Generalização e justificação no estudo das inequações
}

\author{
Joana Mata-Pereira \\ Unidade de Investigação, Instituto de Educação da Universidade de Lisboa \\ joanamatapereira@campus.ul.pt
}

\section{João Pedro da Ponte}

Instituto de Educação da Universidade de Lisboa

jpponte@ie.ul.pt

\begin{abstract}
Resumo
Este artigo analisa os processos de raciocínio matemático de alunos na resolução de tarefas algébricas envolvendo inequações. Esta análise destaca a generalização e a justificação enquanto processos-chave do raciocínio matemático, dando também atenção às representações e processos de significação. Os resultados mostram que na generalização os alunos seguem abordagens indutivas e abdutivas e na justificação baseiam-se em conhecimentos anteriores ou conceitos e propriedades conhecidas. $\mathrm{O}$ uso de diferentes representações parece não limitar o desenvolvimento do raciocínio matemático e os processos de significação surgem intrinsecamente ligados às generalizações ou justificações apresentadas.
\end{abstract}

Palavras-chave: Raciocínio matemático. Generalização. Justificação. Inequações, Álgebra.

\section{Developing mathematical reasoning: Generalization and justification in the study of inequalities}

\begin{abstract}
This article analyses students' mathematical reasoning processes while working on algebraic tasks with inequalities. This analysis emphasizes generalization and justification as key mathematical reasoning processes and also stresses representations and sense-making. The results show that students follow inductive and abductive approaches in generalizing. In justifying, students present arguments based on previous knowledge or known concepts and properties. The use of different representations seems not to constrain the development of mathematical reasoning and sense-making arises intrinsically linked to presented generalizations or justifications.
\end{abstract}

Keywords: Mathematical reasoning. Generalization. Justification. Inequalities. Álgebra. 


\section{Introdução}

Raciocinar, mais do que reproduzir conceitos memorizados e efetuar procedimentos rotineiros, é formular inferências (não imediatas) a partir da informação disponível. Desenvolver a capacidade dos alunos raciocinarem matematicamente é um dos objetivos mais importantes do ensino. Esta capacidade é essencial para se usar eficazmente a Matemática na resolução de problemas nas mais diversas situações e para desenvolver uma compreensão efetiva desta ciência, como conhecimento lógico e coerente (NCTM, 2007). Em Portugal, o programa de Matemática do ensino básico (ME, 2007) apresenta o raciocínio matemático como capacidade transversal a desenvolver ao longo da escolaridade. Na verdade, promover o raciocínio matemático dos alunos constitui um aspeto central do trabalho do professor (LANNIN, ELLIS e ELLIOT, 2011). Contudo, trata-se de um domínio, sobre o qual escasseia a investigação.

Um passo fundamental para promover o desenvolvimento do raciocínio matemático em sala de aula é conhecer melhor como os alunos raciocinam no âmbito desta disciplina. Isso é essencial para desenvolver e avaliar estratégias de ensino que os ajudem a ultrapassar as suas dificuldades e promovam melhores aprendizagens (BABAI, EIDELMAN e STAVY, 2012). Este artigo analisa os processos de raciocínio de alunos do $9 .^{\circ}$ ano $\left(8 .^{\text {a }}\right.$ série) na resolução de tarefas algébricas envolvendo inequações e visa compreender de que modo os processos de raciocínio se relacionam com as representações usadas e com a compreensão de conceitos e procedimentos algébricos.

\section{Raciocínio matemático}

Alguns autores identificam raciocínio matemático com dedução (ALISEDA, 2003; BROUSSEAU e GIBEL, 2005). Nesta perspetiva, raciocínio matemático constitui a realização de inferências lógicas, caracterizadas pela relação necessária entre premissas e conclusões e pela irrefutabilidade das conclusões obtidas (ALISEDA, 2003). No entanto, outros autores alargam o raciocínio matemático ao campo indutivo (LANNIN, ELLIS e ELLIOT, 2011; PÓLYA, 1990), onde se formulam generalizações a partir da identificação de características comuns a diversos casos. Outros, ainda, valorizam o raciocínio abdutivo (RIVERA e BECKER, 2009), em que se formula uma generalização relacionando diversos aspetos de certa situação, que se procuram ajustar como diferentes peças de um puzzle. Assim, podemos dizer que raciocinar matematicamente é usar a informação existente para chegar a novas conclusões por qualquer destes processos, ou seja, fazer inferências de natureza dedutiva, indutiva ou abdutiva. Nestes termos, a capacidade de raciocinar mate- 
maticamente inclui processos como formular questões, formular e testar conjeturas (incluindo generalizações) e realizar justificações.

Formular conjeturas consiste em produzir afirmações que se espera sejam verdadeiras (LANNIN, ELLIS e ELLIOT, 2011). Contudo, em Matemática, mais do que afirmações sobre objetos particulares, fazem-se afirmações gerais sobre classes de objetos, ou seja, generalizações que podem respeitar a propriedades, conceitos ou procedimentos. Formular uma generalização, um processo central no raciocínio matemático, não é mais do que fazer um certo tipo de conjetura de natureza geral. Em situações do dia-a-dia, os alunos estão naturalmente predispostos a realizar generalizações (BECKER e RIVERA, 2005). No entanto, formular uma generalização matemática pertinente representa uma tarefa desafiante (ZAZKIS, LILJEDAHL e CHERNOFF, 2008). Para que os alunos desenvolvam estratégias que conduzam à formulação de generalizações válidas e adequadas, é necessário um trabalho continuado ao longo do seu percurso escolar. Nomeadamente, é necessário que seja promovida e compreendida a transição entre generalizações baseadas maioritariamente em casos particulares e observações empíricas e generalizações baseadas em propriedades ou conceitos matemáticos. Radford (2003) considera três tipos de generalização em Álgebra: (i) factual, aplicando diretamente dados empíricos a novos casos particulares, sem alteração do conjunto de objetos matemáticos; (ii) contextual, alargando os dados empíricos a um novo conjunto de objetos matemáticos; e (iii) simbólica, envolvendo a compreensão e utilização da linguagem algébrica.

Também a justificação, apoiada em procedimentos, propriedades e definições matemáticas (ME, 2007), é central enquanto processo de raciocínio matemático. É necessário, desde os primeiros anos, promover situações que peçam uma justificação alternativa, salientem o que valida uma justificação, enfatizem a explicação do "porquê", levem os alunos a dar sentido a justificações existentes ou redirecionem os alunos para o contexto (BELL, 2011). É ainda necessário que os alunos compreendam e sejam capazes de utilizar justificações que são matematicamente válidas e que, pela sua formulação, contemplam poder matemático (LANNIN, 2005). Como indica este autor, as justificações podem ser agrupadas em cinco níveis de complexidade: (i) não justificar; (ii) apelar à autoridade externa; (iii) utilizar evidência empírica; (iv) utilizar de um exemplo genérico; e (v) justificar dedutivamente.

Para a análise do raciocínio, propomos um quadro conceptual (Figura 1) que relaciona os processos de raciocínio indutivo, abdutivo e dedutivo (zona central da figura) com representações e significação (sense making). Uma vez que é impossível aceder diretamente aos objetos matemáticos, o raciocínio matemático apoia-se necessariamente em representações. Por outro lado, a articulação entre processos de significação e processos de raciocínio são essenciais para uma compreensão efetiva 
da Matemática (NCTM, 2009). Neste quadro, os raciocínios indutivo e abdutivo têm lugar sobretudo durante a formulação de conjeturas e o raciocínio dedutivo ocorre especialmente durante o teste e a justificação.

\section{Significar}

("Sense making")

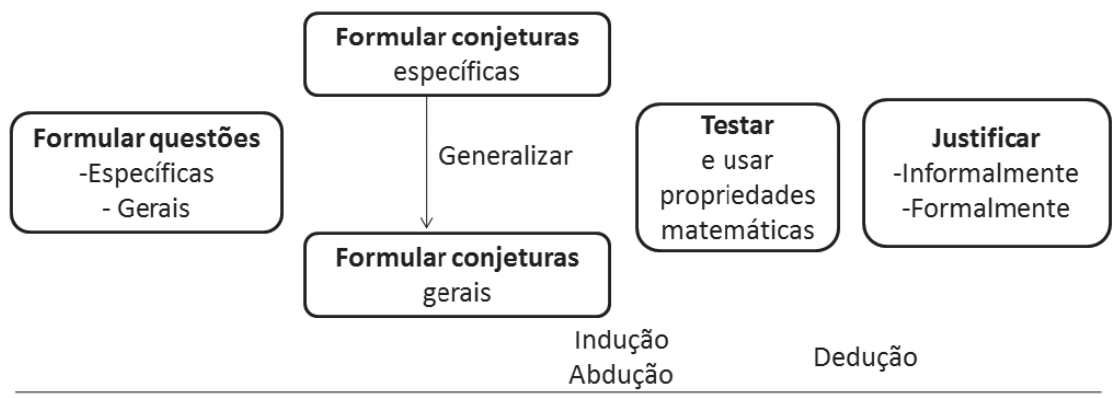

Representar

Linguagem natural, pictórica, algébrica, geométrica, estatística...

\section{Figura 1 - Quadro conceptual para o estudo do raciocínio matemático} (adaptado de MATA-PEREIRA e PONTE, 2011).

$\mathrm{Na}$ atividade matemática, as diferentes representações jogam papéis muitas vezes complementares, assumindo as transformações entre representações um papel central para viabilizar o raciocínio. Duval (2006) apresenta dois tipos de transformações: tratamentos e conversões. Tratamentos são transformações de representações dentro de um mesmo registro, como a resolução algébrica de equações ou a utilização de um cálculo mantendo-se estritamente na mesma notação. Conversões são transformações de representações num certo registro para representações num registro distinto, nomeadamente passar de uma expressão algébrica para a sua representação gráfica ou passar de uma constatação sobre uma relação em linguagem natural para a sua notação algébrica. Estas transformações de um registro para outro são por vezes complexas, mas tornam-se frequentemente essenciais para compreender o objeto matemático em questão.

Os processos de significação também assumem um papel central na compreensão matemática e no desenvolvimento do raciocínio matemático. Estes processos consistem em desenvolver a compreensão de uma situação, contexto ou conceito conectando-o com o conhecimento existente e promovem o desenvolvimento das competências matemáticas dos alunos, tanto a nível dos conhecimentos como dos 
processos (NCTM, 2009). Estando estreitamente relacionados com as conexões, os processos de significação não se restringem a um aspeto do raciocínio, e o próprio raciocínio constitui também um aspeto dos processos de significação. Por um lado, o raciocínio pode basear-se em processos de significação que, através de certas observações, identificam e estabelecem conexões entre elementos comuns. Por outro lado, o raciocínio pode ajudar a compreender o significado do que está a ser observado, ou seja, a ver não só o que é verdade mas também porque é verdade (HANNA, 2000). Assim, organizar o ensino e a aprendizagem enquadrando raciocínio, representações e processos de significação constitui uma condição para a aprendizagem da Matemática com compreensão.

\section{Metodologia de investigação}

O estudo apresentado neste artigo segue uma metodologia de investigação de observação participante (JORGENSEN, 1989), numa abordagem qualitativa e interpretativa (BOGDAN e BIKLEN, 1994), sendo realizado em colaboração com a professora de uma turma de $9 .^{\circ}$ ano $\left(8 .^{a}\right.$ série) composta por dezassete alunos, dos quais, de acordo com a caracterização dada pela professora, sete apresentam baixo desempenho em Matemática, sete médio e três bom. Apesar do fraco desempenho global da turma, esta tem um ambiente de trabalho produtivo, sendo as atividades desenvolvidas maioritariamente em pequenos grupos. Analisamos o raciocínio dos alunos em tarefas sobre inequações. Anteriormente, os alunos já tinham estudado equações do $1 .^{\circ}$ grau, sistemas de duas equações a duas incógnitas e números reais.

A recolha de dados, realizada pela primeira autora (daqui em diante designada por investigadora), teve como local preferencial a sala de aula. A professora da turma lecionou as respetivas aulas e a investigadora acompanhou os alunos na realização das tarefas como "professora de apoio". A recolha de dados foi realizada utilizando processos característicos da observação participante, nomeadamente, observação direta (com gravação áudio e vídeo das aulas) e recolha documental, sendo ainda mantido um diário de bordo com anotações realizadas durante ou logo após as aulas. A análise de dados teve por base o quadro conceptual apresentado na Figura 1, sendo identificados os processos de raciocínio usados, o que os influencia, as representações utilizadas e os processos de significação envolvidos. A organização e interpretação dos dados de acordo com este quadro conceptual permitem uma descrição centrada nos processos de generalização e de justificação. Neste artigo analisamos o trabalho de quatro alunos (cujos nomes são fictícios) em duas questões (Figura 2). 
1. Indica o conjunto solução das seguintes desigualdades, em IR:
1.1. $5+x<10$
1.3. $7<x+1$
1.5. $t-3<0$
1.2. $y-1>-4$
1.4. $2 c<6$
1.6. $\frac{1}{2} x>3$

Princípios de Equivalência para resolver inequações

19 Princípio de Equivalência Quando somamos ou subtraímos o mesmo número a ambos os membros de uma inequação obtemos uma inequação equivalente.

2\% Princípio de Equivalência Se multiplicarmos ou dividirmos ambos os membros de uma inequação pelo mesmo número diferente de zero, obtemos uma inequação equivalente, mantendo-se o sentido da desigualdade se o número for positivo e invertendo o sentido da desigualdade se o número for negativo.

3. Resolve cada uma das seguintes inequações. Sempre que possível representa o conjunto solução sob a forma de intervalo de números reais e na reta real.
3.1. $-3 x<-27$
3.2. $2 x<x+4$
3.3. $-4 x+1 \geq 3-x$

Figura 2 - Questões da tarefa sobre inequações

Antes da questão 1 os alunos tinham resolvido diversas questões envolvendo intervalos de números no conjunto dos números reais e as suas representações habituais. A questão 1 envolve um primeiro contacto dos alunos com inequações, que, neste ponto, não conhecem ainda os princípios de equivalência de inequações. A questão 2, muito semelhante à 1, apresenta algumas convenções referentes às inequações, nomeadamente o que se entende por inequação, solução de uma inequação, membros e termos de uma inequação e inequações equivalentes. A questão 3 envolve pela primeira vez a utilização dos princípios de equivalência de inequações.

\section{Generalização}

A questão 1 proporciona um primeiro contacto com inequações, criando oportunidades para surgirem processos de raciocínio que envolvem generalização. Nomeadamente, durante a sua resolução, espera-se que os alunos generalizem para a resolução de inequações alguns dos procedimentos que já conhecem da resolução de equações. Logo no início, Ana, Débora e Nádia têm alguma dificuldade em interpretar o enunciado, não compreendendo o que é pretendido, pelo que solicitam ajuda. A investigadora, presente junto do grupo, procura esclarecer o que se pretende e acompanha a realização da questão 1.1. Nota-se que, embora as alunas encontrem facilmente valores para $x$, têm alguma dificuldade no conceito de extremo no intervalo de números reais: 
Investigadora: Qual é o número limite?

Ana: Quatro vírgula nove.

Investigadora: (...) Qual é o número limite? Que não me interessa, mas que é o limite.

Nádia: É o cinco.

Investigadora: Portanto é do cinco...

Débora: para trás.

Investigadora: Só que o cinco pertence?

Nádia: Não.

Investigadora: Então o cinco vai ser o quê? É um conjunto, certo?

Débora: Tem a ver com a chaveta, então é a chaveta aberta.

Encontrado o extremo superior, as alunas identificam com alguma facilidade os valores que $x$ pode tomar, reforçando uma ideia já expressa por Débora:

Ana: Do cinco para trás.

(Nádia escreve na sua folha "]5")

Investigadora: É do cinco para trás, então começamos pelo cinco?

Nádia: Não, começamos pelo um...

Investigadora: E não dá abaixo do um?

Ana: Dá, não é menos infinito?

Nádia: Então é de menos infinito... (Figura 3)

Ana: Menos infinito até cinco, chaveta aberta.

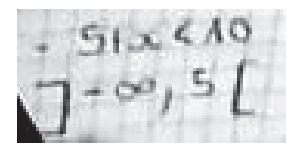

Figura 3 - Resolução de Nádia da questão 1.1.

Esta questão não dá origem a qualquer generalização, mas constitui um ponto de partida para as questões seguintes pois permite às alunas situarem-se perante a noção de relação de ordem, bem como com a identificação de um extremo do intervalo e as representações a utilizar.

Na questão 1.2., a investigadora intervém ajudando as alunas a obterem o intervalo pretendido:

Ana: E este aqui é $y$ menos um é maior que menos quatro.

Débora: Então é... 
Nádia: É menos três. Menos três, menos um.

(...)

Investigadora: Se o menos três é o meu limite, agora têm só de pensar se o que interessa são os números que estão para trás desse ou os números que estão para a frente.

Débora: Estão para trás, é menos infinito com o menos três.

Investigadora: Então experimenta lá menos dois.

Débora: O menos dois...

Nádia: Não, o menos três é o limite. Então é de menos três para lá (gesticula), para dar mais, porque tem cá o sinal de maior.

Ana: Chaveta aberta, menos infinito, menos quatro.

Nádia: Mais infinito. Menos três!

Nesta questão, Nádia, ao dizer "para dar mais, porque tem cá o sinal de maior", enuncia informalmente que, numa desigualdade em que $a+x>b+c$ ( $a, b, c$ constantes) o conjunto solução é da forma $] k$, $+\infty[$. Esta generalização, ainda que apenas seja válida para $x>0$, permite identificar de imediato se o caso da igualdade dos membros corresponde ao extremo inferior ou superior do intervalo de números reais.

Na questão 1.3., Ana, Débora e Nádia conseguem utilizar parcialmente as ideias desenvolvidas nas questões anteriores e aplicá-las à nova situação:

Débora: O sete é menor que $x$ mais um.

Nádia: Portanto o limite é o seis.

Investigadora: O limite é o seis, mas é para trás ou para a frente?

Nádia: É para trás.

Investigadora: Se for... Experimentem outro.

(...)

Nádia: O cinco mais um vai dar seis.

Investigadora: E sete é menor do que seis?

Débora: Não.

(...)

Nádia: São do seis para a frente. O sete mais um vai dar oito, é maior que sete. Ana: Fica mais infinito.

Nádia: E é aberto.

Ana: Fica seis para mais infinito (escreve o intervalo de números reais ]6, $+\infty[$ ). 
A ideia de "número limite" usada pela investigadora na conversa com as alunas na questão 1.1. é facilmente utilizada por elas na resolução das questões 1.2. e 1.3., levando-as a encontrar facilmente um dos extremos do intervalo. Contudo, as alunas mostram dificuldades em concluir se se trata do extremo superior ou inferior do intervalo de números reais ao qual $x$ pertence. Tratando-se do seu primeiro contacto com inequações, a identificação imediata dos extremos “- 3 " e " 6 ", respetivamente nas questões 1.2. e 1.3., pressupõe, implicitamente, uma generalização relativa à utilização da igualdade entre os membros para obter um dos extremos do intervalo. Assim, as alunas generalizam para a resolução de inequações um procedimento já conhecido para a resolução de equações, ao transformarem implicitamente a inequação numa equação. Esta generalização baseia-se no caso particular usado na questão 1.1., para o qual as alunas apenas conseguem utilizar o conceito de extremo de um intervalo com alguma intervenção da investigadora. Note-se que, na resolução da inequação da questão 1.3., as alunas não aplicam a generalização feita por Nádia na questão 1.2. apesar desta permitir determinar se o extremo identificado corresponde ao extremo inferior ou superior do conjunto solução.

Ao longo da resolução da questão 1, Ana, Débora e Nádia realizam, sem dificuldades, conversões entre a linguagem simbólica com variáveis e a linguagem natural oral, procurando assim interpretar (no sentido de "dizer por palavras suas" como referem Bishop e Gofree, 1986) o enunciado de cada subquestão. Utilizam essencialmente a linguagem natural oral, realizando a conversão para a linguagem simbólica sem variáveis para as respostas de cada subquestão. Na resolução desta questão, as alunas estabelecem conexões entre as inequações apresentadas e as igualdades entre os membros, correspondente a equações. Contudo, após estabelecerem esta conexão, mostram dificuldades na conexão entre a inequação e os valores possíveis para $x$, o que inviabiliza a construção do intervalo de números reais ao qual $x$ pertence. Assim, as alunas aplicam a generalização obtida implicitamente para encontrar um dos extremos do intervalo, mas não conseguem aplicar a generalização enunciada por Nádia, que lhes permite compreender de imediato se o valor encontrado se trata do extremo inferior ou superior. A falha na aplicação desta generalização leva à necessidade de teste de casos particulares para identificar se o limite obtido é o extremo inferior ou superior do intervalo de números reais a construir.

\section{Justificação}

Na questão 3, Afonso começa por afirmar que já aprendeu a resolver inequações, pelo que a investigadora lhe solicita que apresente a sua resolução: 
Investigadora: É assim? Deixa lá ver o que é que fizeste. Dividiste por... Afonso: Três.

Investigadora: Mas mudaste aqui uns sinais...

Afonso: Foi ela que disse que era assim.

Investigadora: Quem é que disse?

Afonso: Filó!

Ainda que o resultado esteja correto, o aluno não mostra ter compreendido como resolver a inequação, limitando-se a reproduzir a resposta dada por uma colega. A investigadora sugere-lhe então que utilize os princípios de equivalência enunciados na tarefa:

Investigadora: Lê lá aqui este, o segundo princípio de equivalência.

Afonso: (responde antes de ler) Então, mas é por causa disso! (...) (lê o princípio em silêncio) Então tenho de inverter o símbolo.

Investigadora: Porque...

Afonso: Estou a dividir por um número negativo.

Investigadora: Os princípios são muito parecidos com os das equações.

Afonso: Mentira, as equações não têm isto.

(...)

Investigadora: $x$ maior que nove, como é que eu represento na forma de intervalo?

Afonso: Então, fica... (Figura 4).

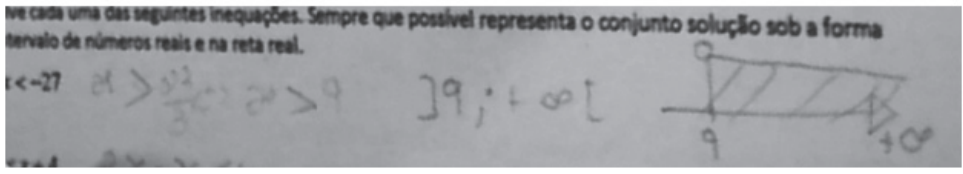

Figura 4 - Resolução de Afonso da questão 3.1.

Nesta questão Afonso utiliza tratamentos na linguagem simbólica com variáveis, ainda que na sua primeira resolução não consiga fazer os tratamentos necessários à obtenção do resultado pretendido. De seguida, consegue realizar os tratamentos necessários em linguagem simbólica com variáveis, articulando estes tratamentos com a linguagem natural escrita presente na tarefa. Na fase final da sua resolução, o aluno converte com facilidade a condição obtida em linguagem simbólica com variáveis tanto para a representação em forma de intervalo como para a representação na reta numérica. 
Na primeira fase da sua resolução, Afonso, quando interpelado, justifica que a alteração de sinais é realizada por indicação de uma colega, baseando portanto a sua justificação numa autoridade externa. Deste modo, parece não estabelecer as conexões necessárias à resolução da inequação, não mostrando compreender a situação. Numa segunda fase, o aluno consegue articular a resolução da inequação com a informação referente aos princípios de equivalência, estabelecendo as conexões necessárias à compreensão da situação. É ainda induzido a estabelecer uma relação entre os princípios de equivalência das equações e os princípios de equivalência das inequações, conseguindo destacar de imediato a diferença nas alterações da desigualdade. Quando lhe é solicitado que justifique a alteração do sentido da desigualdade, utiliza o princípio de equivalência enunciado, mostrando compreendê-lo e, consequentemente, apresentando uma justificação válida.

Na questão 3.2. a investigadora solicita a justificação de cada passo da resolução. Contudo, Afonso só o faz para o primeiro passo, atendendo a que o passo seguinte (e último) na resolução da inequação é de complexidade reduzida:

Investigadora: A minha pergunta é, tu passaste o $x$ para o outro lado, e a minha pergunta é, porque é que...

Afonso: Para isolar a incógnita.

Investigadora: Mas a minha pergunta é, porque é que tu podes passar o $x$ para o outro lado.

Afonso: Porque me disseram que podia.

Nesta primeira fase da resolução, Afonso realiza tratamentos em linguagem simbólica com variáveis e utiliza a linguagem natural oral para as suas justificações. Após esta indicação, o aluno realiza com facilidade os tratamentos necessários à conclusão da resolução, utilizando, como anteriormente, a linguagem simbólica com variáveis. Na sua justificação referente à mudança de $x$ para o primeiro membro da inequação, o aluno remete para uma autoridade indefinida, o que não constitui um argumento matematicamente válido, invalidando a sua justificação. Contudo, estabelece conexões entre a resolução de equações e a resolução de inequações, utilizando procedimentos análogos aos já conhecidos das equações.

Nesta questão salienta-se ainda que Afonso, quando é questionado a primeira vez, interpreta erradamente a questão da investigadora mas refere que a alteração dos sinais implica a alteração do sinal da desigualdade, referindo-se implicitamente à multiplicação de ambos os membros da inequação por -1: 
Investigadora: Porque é que tu podes mudar o $x$ para o outro lado?

Afonso: Então, posso meter o quatro negativo, o $2 x$ negativo e o $x$ positivo e mudar o símbolo.

Esta afirmação, mesmo não sendo solicitada para a questão 3.2., apresenta evidência de alguma compreensão do segundo princípio de equivalência apresentado na tarefa.

\section{Conclusão}

$\mathrm{Na}$ formulação de generalizações apresentada, as alunas seguem uma abordagem indutiva, generalizando as relações observadas num caso particular para uma classe de objetos mais ampla. No entanto, Nádia, ao formular uma nova generalização considera outros aspetos da situação, não se limitando a considerar o caso particular da questão 1.1., o que remete para uma abordagem abdutiva.

No que respeita à justificação, os alunos não dão relevância às características necessárias para que seja matematicamente válida, apresentando tanto justificações válidas como justificações não válidas. Estas últimas, tal como evidenciado por Lannin (2005), surgem por vezes associadas a uma referência a uma autoridade externa, como na justificação de Afonso da questão 3.2. Esta justificação apresentada pelo aluno remete para uma aplicação meramente rotineira de procedimentos algébricos, o que limita a sua compreensão da situação e dificulta a apresentação de uma justificação válida. Esta situação, semelhante às destacadas por Henriques (2010), remete para um desenvolvimento instável do sentido de símbolo, necessário ao raciocínio algébrico e consequentemente à capacidade de estabelecer conexões. Neste estudo, os alunos apresentam também justificações válidas para as questões apresentadas ou para situações que surgem na discussão das questões com os colegas. Em parte destas situações, os alunos baseiam-se em conhecimentos anteriores ou argumentos válidos para a situação em questão, nomeadamente conceitos ou propriedades conhecidas.

No que respeita às representações, os alunos utilizam a linguagem natural oral e escrita e a linguagem simbólica sem e com variáveis, não apresentando dificuldades significativas nas transformações, sejam tratamentos ou conversões. Assim, e considerando a facilidade com que os alunos realizam transformações entre representações, a utilização de diferentes representações não parece limitar o desenvolvimento do raciocínio matemático dos alunos. Quanto aos processos de significação, em grande parte das situações apresentadas os alunos conseguem estabelecer conexões entre os aspetos da questão e as propriedades ou conceitos necessários à sua resolução, manifestando compreensão da situação. Contudo, em alguns casos, não estabelecem 
todas as conexões necessárias, o que sugere uma compreensão apenas parcial da situação em questão. Quando os alunos são direcionados, através do questionamento, para estabelecerem as conexões em falta, aparentam desenvolver os seus processos de significação promovendo uma compreensão mais aprofundada das questões. Deste modo, os processos de significação surgem intrinsecamente ligados às generalizações ou justificações apresentadas na medida em que, quando há dificuldades nas conexões entre os conceitos e propriedades necessários à consecução da tarefa, parece igualmente existir uma dificuldade na generalização ou na justificação.

Nas relações estabelecidas entre raciocínio, representações e significação, o modelo de análise utilizado (Figura 1) revelou-se um instrumento útil pois mostra como alguns alunos são capazes de realizar certas generalizações e justificações, utilizando raciocínio indutivo, abdutivo e dedutivo e como articulam os seus processos de raciocínio com processos de significação e o uso de representações. A análise apresentada contribui para o conhecimento dos processos de raciocínio dos alunos. Elaborar tarefas que contribuam para o desenvolvimento destes processos e investigar modos de exploração na sala de aula constitui um importante desafio para trabalho futuro em educação matemática.

\section{Referências}

ALISEDA, A. Mathematical reasoning vs. abductive reasoning: A structural approach. Synthese, v. 134, p. 25-44, 2003.

BABAI, R., EIDELMAN, R., \& STAVY, R. Preactivation of inhibitory control mechanisms hinders intuitive reasoning. International Journal of Science and Mathematics Education, v.10, p. 763-775, 2012.

BECKER, R., \& RIVERA, F. Generalization strategies of beginning high school algebra students. In H. Chick \& J. Vincent (Eds.), Proceedings of the 29th Conference of the International Group for the Psychology of Mathematics Education. Melbourne: PME, 2005. v. 4, p. 121-128.

BELL, C. Proofs without words: A visual application of reasoning and proof. Mathematics Teacher, v. 104, p. 690-695. 2011.

BISHOP, A., \& GOFFREE, F. Classroom organization and dynamics. In B. Christiansen, A. G. Howson \& M. Otte (Eds.), Perspectives on mathematics education. Dordrecht: Reidel, 1986. p. 309-36. 
BOGDAN, R., \& BIKLEN, S. Investigação qualitativa em educação. Porto: Porto Editora, 1994.

BROUSSEAU, G., \& GIBEL, P. Didactical handling of students' reasoning processes in problem solving situations. Educational Studies in Mathematics, v. 59, p. 13-58, 2005.

DUVAL, R. A Cognitive analysis of problems of comprehension in a learning of mathematics. Educational Studies in Mathematics, v. 61, p. 103-131, 2006.

HANNA, G. Proof and its classroom role: a survey. In Actas do IX EIEM - Encontro de Investigação em Educação Matemática. Fundão: SPCE-SEM, 2000. p. 75-104.

HENRIQUES, A. O pensamento matemático avançado e a aprendizagem da análise numérica no contexto de actividades de investigação. Tese de Doutoramento, Universidade de Lisboa, Lisboa, 2010.

JORGENSEN, D. L. Participant observation. Newbury Park, CA: Sage, 1989.

LANNIN, J. Generalization and justification: The challenge of introducing algebraic reasoning through patterning activities. Mathematical thinking and learning, v. 7, p. 231-258, 2005.

LANNIN, J., ELLIS A. B., \& ELLIOT, R. Developing essential understanding of mathematics reasoning for teaching mathematics in prekindergarten-grade 8. Reston, VA: NCTM, 2011.

MATA-PEREIRA, J., \& PONTE, J. P. Raciocínio matemático em contexto algébrico: Uma análise com alunos do 9. ${ }^{\circ}$ ano. In EIEM 2011 - Atas do Encontro de Investigação em Educação Matemática, 2011. p. 347-364.

ME. Programa de Matemática do ensino básico. Lisboa: Ministério da Educação/ DGIDC, 2007.

NCTM. Princípios e Normas para a Matemática Escolar. Lisboa: APM, 2007. 
NCTM. Focus in high school mathematics: Reasoning and sense making. Reston, VA: NCTM, 2009.

OLIVEIRA, P. O raciocínio matemático à luz de uma epistemologia soft. Educação e Matemática, v. 100, p. 3-9, 2008.

PÓLYA, G. Mathematics and plausible reasoning (ed. original 1954), Vol. 1 e 2. Princeton, NJ: Princeton University Press, 1990.

RADFORD, L. Gestures, speech, and the sprouting of signs: A semiotic-cultural approach to students' types of generalization. Mathematical Thinking and Learning, v. 5, p. 37-70, 2003.

RIVERA, F., \& BECKER, J. Algebraic reasoning through patterns. Mathematics Teaching in the Middle School, v. 15, p. 213-221, 2009.

ZAZKIS, R., LILJEDAHL, P., \& CHERNOFF, E. The role of examples in forming and refuting generalizations. ZDM Mathematics Education, v. 40, p. 131-141, 2008.

Submetido em dezembro de 2012 Aprovado em janeiro de 2013 